\title{
Cooperativity, absolute interaction, and algebraic optimization
}

\author{
Nidhi Kaihnsa ${ }^{1} \cdot{\text { Yue } \text { Ren }^{2} \text { (D) } \cdot \text { Mohab Safey El Din }}^{3} \cdot$ Johannes W. R. Martini ${ }^{4}$
}

Received: 30 July 2019 / Revised: 4 May 2020 / Published online: 23 September 2020

(C) The Author(s) 2020

\begin{abstract}
We consider a measure of cooperativity based on the minimal interaction required to generate an observed titration behavior. We describe the corresponding algebraic optimization problem and show how it can be solved using the nonlinear algebra tool SCIP. Moreover, we compute the minimal interactions and minimal molecules for several binding polynomials that describe the oxygen binding of various hemoglobins under different conditions. We compare their minimal interaction with the maximal slope of the Hill plot, and discuss similarities and discrepancies with a view towards the shapes of the binding curves.
\end{abstract}

Mathematics Subject Classification 92C40 - 90C23

Yue Ren

yue.ren@swansea.ac.uk

https://yueren.de

Nidhi Kaihnsa

nidhi_kaihnsa@brown.edu

https://sites.google.com/view/kaihnsa/

Mohab Safey El Din

mohab.safey@lip6.fr

https://www-polsys.lip6.fr/ safey/

Johannes W. R. Martini

jwrmartini@gmail.com

1 Division of Applied Mathematics, Brown University, 182 George Street, Providence, RI 02912, USA

2 Department of Mathematics, Swansea University, Swansea University Bay Campus, Swansea SA1 8EN, UK

3 Laboratoire d'Informatique de Paris 6, LIP6, Équipe PolSys, Sorbonne Université, CNRS, Paris, France

4 International Maize and Wheat Improvement Center, Texcoco, Mexico 


\section{Introduction}

Interaction between components is a fundamental feature of biological systems. While a simple system of independent subunits is completely defined by its subunits, a complex system with interactions is more than the sum of its parts. A classical example of a small biological system with non-trivial interaction is hemoglobin with its four binding sites for oxygen (Bohr et al. 1904; Barcroft 1913; Hill 1913). The ligand oxygen binds to the four binding sites of the (target) molecule hemoglobin and the interaction can be seen on the overall (isotherm) binding curve, which relates the average ligand saturation inside the system to the ligand concentration outside the system at constant temperature (Hill 1985). While the binding curve of an independent system is linear, the binding curve of hemoglobin has a sigmoidal shape (Barcroft 1913; Hill 1913), see Fig. 1.

The sigmoidal shape implies that the extremal states of full and zero saturation are more stable than the intermediate states of partial saturation. This phenomenon is commonly referred to as cooperativity, named after the intuition that bound ligands affect, either positively or negatively, the chances of new ligands binding to the still open sites (see Stefan and Le Novère 2013 for a review and interpretations). Cooperativity is ubiquitous in nature. It is an essential trait for transport molecules, and its importance in the formation of multi-protein complexes (Roy et al. 2017), in general signal transduction processes (Salakhieva et al. 2016; Lenaerts et al. 2009; Martini et al. 2016b) and in the regulation of noise (Gutierrez et al. 2009; Monteoliva et al. 2013) has been discussed.

When investigating cooperativity, one interesting problem is to connect macroscopic behavior to microscopic behavior. Here, macroscopic refers to the measured average saturation at varying ligand concentration, and microscopic refers to which individual sites are saturated and how the different sites interact. An example is the aforementioned interpretation of occupied sites increasing the probability of ligands

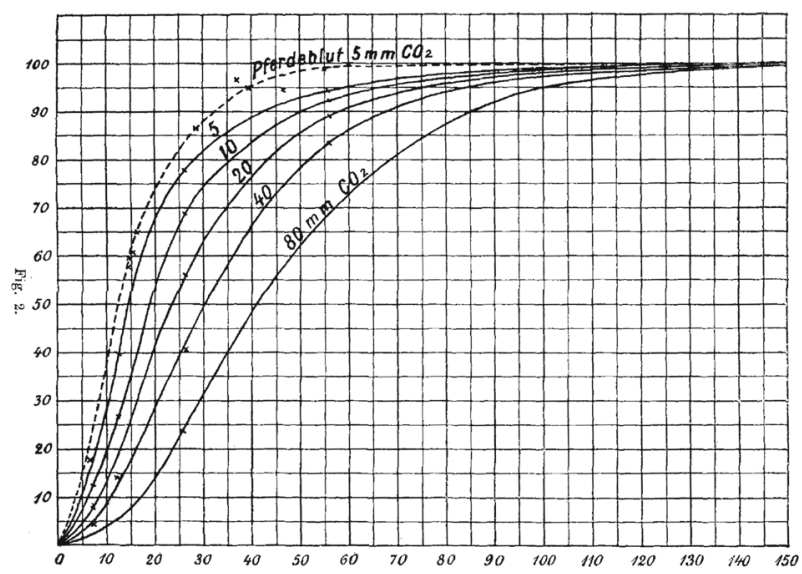

Fig. 1 The oxygen concentration in dog and horse blood in relation to the ambient oxygen concentration (Bohr et al. 1904, Fig. 2) 
binding to unoccupied sites (Stefan and Le Novère 2013; Onufriev and Ullmann 2004). Ideally, such connections between microscopic and macroscopic behavior provide insight on the potential structure and the inner workings of the molecule.

However, the overall binding curve alone does not allow for much inference on the individual site binding curves, similar to how a large sum allows for little inference on its summands. Given a fixed overall binding curve, there generally is no unique molecule, defined by binding and interaction energies, with the prescribed macroscopic binding behavior, but infinitely many. Thus many works feature additional constraints which reduce the ambiguity, such as considering only small system with one or two binding sites (Hunter and Anderson 2009) or symmetric systems with identical binding sites (Abeliovich 2016; Rong et al. 2019). These constraints have the added benefit of simplifying the mathematical problem, allowing for easier inference on interaction energy terms, conditional probabilities, correlations or other quantities. Ideally, constraints should be motivated by knowledge on the chemical structure of the molecule.

A biologically and mathematically interesting question is for instance whether there exist a molecule with independent sites for a fixed overall binding curve. Assuming evolutionary pressure on the transport properties of hemoglobin, it should not be possible to model the system's overall binding curve through an independent system, since otherwise the tetrameric structure of hemoglobin would hold no advantage over four monomers.

The decoupled sites representation (Onufriev et al. 2001; Martini and Ullmann 2013) is a central theorem in this context, which states that every overall binding curve can be generated by a unique hypothetical molecule with possibly non-real binding energies and independent binding sites. The binding energies are determined by the roots of the binding polynomial of the overall binding curve. In particular, the realness of the roots show whether interaction is necessary to achieve the overall binding behavior and thus provide a qualitative definition of cooperativity.

In case interaction is required to generate the overall binding curve, a natural question is how to quantify the degree of cooperativity. This is classically done using the maximal slope of the Hill plot, which is the plot of binding curve on a logarithmic $\mathrm{x}$ axis (see Fig. 6). A new approach (Martini 2017) is to look at the minimal interaction required to generate the considered overall binding curve. The minimal interaction quantifies the minimal deviation from an independent system that is necessary to explain the observed overall binding behavior.

The manuscript in hand studies the problem of finding molecules generating a given overall binding curve with minimal interaction. We show that looking for the minimal interaction energy required to generate a given overall titration behavior, leads to an algebraic optimization problem which can be partially tackled using software such as SCIP (Gleixner et al. 2018). We calculate the minimal molecules of various binding curves in existing datasets (Connelly et al. 1986; Ikeda-Saito et al. 1983; Imai 1973). These binding curves describe the oxygen binding of hemoglobins under different chemical treatments and under different temperatures. We compare their minimal interaction with the maximal slope of the Hill plot, and discuss similarities and discrepancies with a view towards the shapes of the binding curves. 


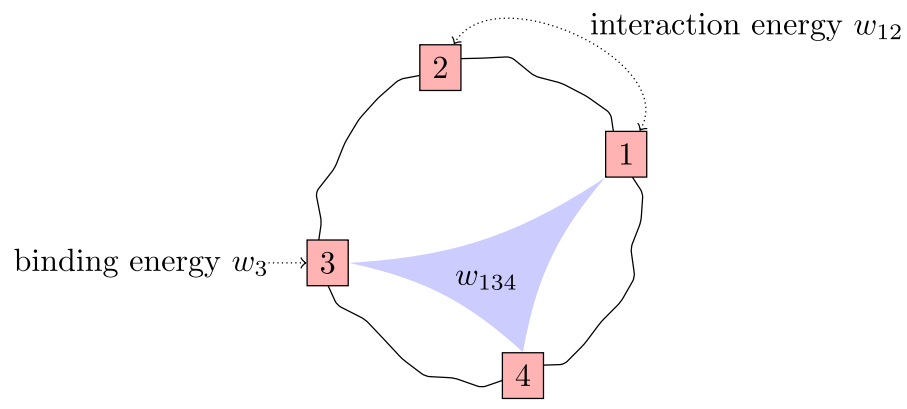

Fig. 2 A molecule with 4 binding sites

\section{Recapitulation of the mathematical framework}

In this section, we briefly review the model for ligand binding based on the grand canonical ensemble of statistical mechanics and recall the notion of minimal absolute interaction from (Martini 2017). We refer the reader to (Ben-Naim 2013; Wyman and Gill 1990; Hill 1985) for detailed expositions on the model.

Definition 2.1 A hypothetical molecule $\mathrm{W}$ with $n$ sites is a complex point, whose coordinates are indexed by the subsets of $[n]:=\{1, \ldots, n\}$ and with $w_{\emptyset}=1$ :

$$
\mathrm{W}=\left(w_{I}\right)_{I \subseteq[n]} \in \mathbb{C}^{2^{n}} .
$$

A (real) molecule is a hypothetical molecule whose coordinates are real and positive.

We refer to $w_{I}$ as binding energies if $|I|=1$ and interaction energies if $|I|>1$. For the sake of brevity, we abbreviate $w_{\left\{i_{1}, \ldots, i_{r}\right\}}$ to $w_{i_{1} \ldots i_{r}}$ for $i_{1}<\cdots<i_{r}$ (see Fig. 2).

Note that we refer to $w_{i_{1} \ldots i_{r}}$ as energy even though it is actually representing the exponential of an energy difference (Ben-Naim 2013; Wyman and Gill 1990; Schellman 1975). To be precise:

- The binding energy $w_{i}$ encodes the difference between the free energies of the completely unsaturated state and the state with a single ligand bound to site $i$.

- The interaction energy $w_{i_{1} i_{2}}$ encodes the difference between the free energy when sites $i_{1}, i_{2}$ are occupied and the sum of the energies when either $i_{1}$ or $i_{2}$ is occupied.

- Analogously, $w_{i_{1} \ldots i_{r}}$ encodes the difference between the free energy when sites $i_{1}, \ldots, i_{r}$ are occupied and the sum of all energies when a proper subset of $i_{1}, \ldots, i_{r}$ are occupied.

As such, the only "sensible" values for $w_{i_{1} \ldots i_{r}}$ are positive real numbers. However, mathematically it is easier to work with hypothetical molecules, and they allow for interesting phenomena such as the decoupled sites representation (Onufriev et al. 2001; Martini and Ullmann 2013). In this article, we will mainly focus on real molecules. 
Definition 2.2 The overall binding curve of a real molecule $\mathrm{W}=\left(w_{I}\right)_{I \subseteq[n]} \in\left(\mathbb{R}_{>0}\right)^{2^{n}}$ with $n$ sites is defined as

$$
\Psi(\Lambda)=\frac{n a_{n} \Lambda^{n}+(n-1) a_{n-1} \Lambda^{n-1}+\cdots+a_{1}}{a_{n} \Lambda^{n}+a_{n-1} \Lambda^{n-1}+\cdots+a_{1}+a_{0}},
$$

also commonly known as the Adair equation (Adair et al. 1925; Stefan and Le Novère 2013), with positive real coefficients

$$
a_{k}:=\sum_{|I|=k} \prod_{I^{\prime} \subseteq I} w_{I^{\prime}} \in \mathbb{R}_{>0} .
$$

The binding polynomial $\Phi(\mathrm{W})$ is the univariate polynomial of degree $n$ in its denominator,

$$
\Phi(\mathrm{W}):=a_{n} \Lambda^{n}+\cdots+a_{1} \Lambda+a_{0} .
$$

The binding polynomial $\Phi$ uniquely determines the overall binding curve $\Psi$.

Example 2.3 Let $\mathrm{W}=\left(w_{I}\right)_{I \subseteq[3]} \in\left(\mathbb{R}_{>0}\right)^{2^{3}}$ be a molecule with 3 sites. The binding polynomial $\Phi(\mathrm{W})=a_{3} \Lambda^{3}+a_{2} \Lambda^{2}+a_{1} \Lambda+a_{0}$ is a real univariate polynomial of degree 3 whose coefficients are given by

$$
\begin{aligned}
& a_{0}=w_{\emptyset}=1, \\
& a_{1}=w_{1}+w_{2}+w_{3}, \\
& a_{2}=w_{1} w_{2} w_{12}+w_{1} w_{3} w_{13}+w_{2} w_{3} w_{23}, \\
& a_{3}=w_{1} w_{2} w_{3} w_{12} w_{13} w_{23} w_{123} .
\end{aligned}
$$

Note that two different molecules may have the same binding polynomial and thus the same binding curve, e.g.,

$$
\begin{aligned}
& \mathrm{W}=\left(\begin{array}{cccccccccc}
w_{\emptyset} & w_{1} & w_{2} & w_{3} & w_{12} & w_{13} & w_{23} & w_{123} \\
1 & 3 & 1 & 1 & 1 & 1 & 2 & 2
\end{array}\right), \\
& \mathrm{W}^{\prime}=\left(\begin{array}{llllllll}
1 & 2 & 2 & 1 & 1 & 1 & 1 & 3
\end{array}\right) .
\end{aligned}
$$

Hence, the map $\Phi$ which maps a molecule with $n$ sites to its binding polynomial is not injective.

Computing hypothetical or real molecules $\mathrm{W}$ with a fixed binding polynomial $P$ can be a hard task, though there are well established algorithms and software for both. Let $\mathcal{M}_{\mathbb{C}}(P)$ resp. $\mathcal{M}_{\mathbb{R}}(P)$ denote the set of hypothetical resp. real molecules $\mathrm{W}$ with $\Phi(\mathrm{W})=P$. Then $\mathcal{M}_{\mathbb{C}}(P)$ is a algebraic variety, i.e., a set of complex points given by polynomial equations, while $\mathcal{M}_{\mathbb{R}}(P)$ is a semialgebraic set, i.e., a set of real points given by polynomial equations and inequalities.

Computing a hypothetical molecule in $\mathcal{M}_{\mathbb{C}}(P)$ is generally easier than $\mathcal{M}_{\mathbb{R}}(P)$. It can be done purely numerically, e.g., using numerical algebraic geometry and software 
such as BERTINI, PHCPACK, HOM4PS or JULIAHOMOTOPYCONTINUATION. Computing a real molecule in $\mathcal{M}_{\mathbb{R}}(P)$ is a significantly more involved task. There are many approaches to it and most require some symbolic computation, such as RAGLIB which uses a mix of symbolic and numerical computations.

However, both $\mathcal{M}_{\mathbb{C}}(P)$ and $\mathcal{M}_{\mathbb{R}}(P)$ are of high dimension and degree (Ren et al. 2019), so that it is unclear what information can be read off a randomly computed molecule. Rather, we need sensible constraints which gives us molecules of interest. Since the aim of this paper is to study cooperativity in terms of the interaction between the binding sites, we introduce the notions of absolute interaction and minimal interaction:

Definition 2.4 The (absolute) interaction of a molecule $\mathrm{W}=\left(w_{I}\right) \in\left(\mathbb{R}_{>0}\right)^{2^{n}}$ is given by

$$
\|\mathrm{W}\|:=\prod_{|I|>1} \max \left(w_{I}, w_{I}^{-1}\right)
$$

Since $w_{I}$ represents the exponential of a difference of actual energies, which can be positive or negative, $\max \left(w_{I}, w_{I}^{-1}\right)$ represents the exponential of the absolute value of that difference. Hence, the absolute interaction $\|\mathrm{W}\|$ represents the exponential of the sum of the absolute values of all energy differences. The minimal value $\|\mathrm{W}\|$ can adopt is 1 which corresponds to a minimal physical interaction energy of 0 .

Definition 2.5 Given a binding polynomial $P$ of degree $n$, the minimal interaction is

$$
\|P\|:=\min \left\{\|\mathrm{W}\| \mid \mathrm{W} \in\left(\mathbb{R}_{>0}\right)^{2^{n}} \text { with } \Phi(\mathrm{W})=P\right\} .
$$

We call a molecule $W$ minimal, if $\|W\|=\|\Phi(W)\|$.

In other words, the minimal interaction $\|P\|$ is the minimal interaction required to generated the binding polynomial $P$. Thus it quantifies the minimal deviation from an independent system that is required to explain the observed overall binding behavior. The following proposition states that the minimum exists.

Proposition 2.6 (Martini 2017, §4) For any univariate polynomial P of degree $n$ with positive coefficients there exists a molecule $\mathrm{W} \in\left(\mathbb{R}_{>0}\right)^{2^{n}}$ with $n$ sites such that $\Phi(\mathrm{W})=P$ and $\|\mathrm{W}\|=\|P\|$.

Since cooperativity is a property of the binding curve which emerges from the interaction between the sites, the minimal interaction of a binding polynomial is a natural candidate for quantifying cooperativity. In addition, (Martini 2017, §4) shows that it has several properties that are desirable of a quantifier.

Example 2.7 Consider the following binding polynomials:

$$
\begin{aligned}
& P_{1}:=4 \Lambda^{3}+3 \Lambda^{2}+2 \Lambda+1, \\
& P_{2}:=6 \Lambda^{3}+7 \Lambda^{2}+4 \Lambda+1=(2 \Lambda+1)\left(3 \Lambda^{2}+2 \Lambda+1\right), \\
& P_{3}:=6 \Lambda^{3}+11 \Lambda^{2}+6 \Lambda+1=(\Lambda+1)(2 \Lambda+1)(3 \Lambda+1) .
\end{aligned}
$$



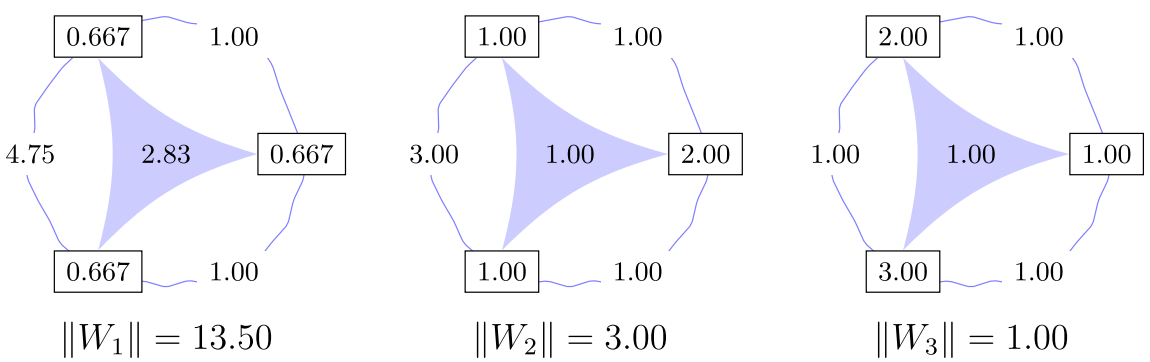

Fig. 3 Minimal molecules for binding polynomial $P_{1}, P_{2}, P_{3}$

A brief computation reveals that the minimal absolute interactions are

$$
\left\|P_{1}\right\|=13.50, \quad\left\|P_{2}\right\|=3, \quad\left\|P_{3}\right\|=1 .
$$

The computation for $\left\|P_{1}\right\|$ is explicitly shown in Example 3.3. Figure 3 illustrates the minimal molecules for each binding polynomial. Since $P_{3}$ factorizes into three real linear factors, its minimal molecule has only trivial interactions.

\section{Computing the minimal interaction}

In this section, we consider the problem of computing the minimal absolute interaction for a given binding polynomial $P$ :

$$
\begin{array}{ll}
\underset{\mathrm{W}}{\operatorname{minimize}} & \|\mathrm{W}\| \\
\text { subject to } & \Phi(\mathrm{W})=P
\end{array}
$$

For the sake of clarity, we will restrict ourselves to the case of molecules with 4 binding sites, though our arguments also apply to the general case. We will discuss what makes Problem (1) challenging, and what can or cannot be done towards solving it from a practical point of view.

\subsection{The initial problem}

Problem (1) is commonly called the standard form of a continuous optimization problem with $\|W\|$ being the objective function and $\Phi(\mathrm{W})=P$ the constraints. The set of all molecules $W$ which satisfy the constraints is the feasible set.

Generally speaking, there are many approaches for solving optimization problems depending on the type of the objective function and the constraints, which may also include inequalities. If both are linear, the problem is also known as a linear program. If the objective function is quadratic and the constraints are linear, the problem is also called a quadratic program. Both types of optimization problems are well-studied with plenty of literature and software solutions. If both objective function and constraints 
are polynomial, the problem is a polynomial optimization problem or an algebraic optimization problem. This is the type of problem that needs to be solved for Problem 1 , and solving it generally involves using sums of squares approaches (Lasserre 2001; Parrilo 2003) or critical point methods (Greuet and Safey El Din 2014).

For molecules with 4 binding sites specifically, Problem (1) is of the following form for a given binding polynomial $P=a_{4} \Lambda^{4}+a_{3} \Lambda^{3}+a_{2} \Lambda^{2}+a_{1} \Lambda$ :

$$
\begin{aligned}
& \underset{\left(w_{I}\right)_{I \subseteq[4]}}{\operatorname{minimize}} \prod_{|I|>1} \max \left(w_{I}, w_{I}^{-1}\right) \\
& \text { subject to } a_{1}=w_{1}+w_{2}+w_{3}+w_{4} \text {, } \\
& a_{2}=w_{1} w_{2} w_{12}+w_{1} w_{3} w_{13}+w_{1} w_{4} w_{14} \\
& +w_{2} w_{3} w_{23}+w_{2} w_{4} w_{24}+w_{3} w_{4} w_{34} \text {, } \\
& a_{3}=w_{1} w_{2} w_{3} w_{12} w_{13} w_{23} w_{123}+w_{1} w_{2} w_{4} w_{12} w_{14} w_{24} w_{124} \\
& +w_{1} w_{3} w_{4} w_{13} w_{14} w_{34} w_{134}+w_{2} w_{3} w_{4} w_{23} w_{24} w_{34} w_{234} \text {, } \\
& a_{4}=w_{1} w_{2} w_{3} w_{4} w_{12} w_{13} w_{14} w_{23} w_{24} w_{34} w_{123} w_{124} w_{134} w_{234} w_{1234} \text {. }
\end{aligned}
$$

Problem (2) is extremely hard to solve because of two reasons:

- the objective function contains 11 maxima,

- the constraints contain polynomials of degree up to 15 .

In the following, we will briefly discuss the two challenges separately.

\subsection{Resolving the maxima in the objective function}

The maxima in the objective function of Problem (2) can be resolved in two ways.

One standard way is to reformulate the objective function as a maximum of polynomial functions and linearize the objective function through additional constraints: Abbreviating $\left(\begin{array}{c}{[4]} \\ >1\end{array}\right):=\{I \subseteq[4]|| I \mid>1\}$, we define for each set of interactions $\mathcal{I} \subseteq\left(\begin{array}{c}{[4]} \\ >1\end{array}\right)$

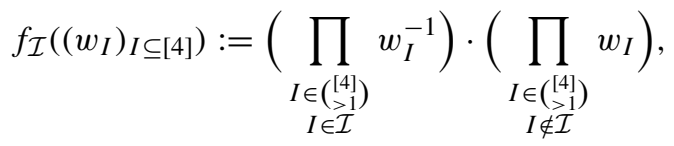

so that

$$
\left\|\left(w_{I}\right)_{I \subseteq[4]}\right\|=\max _{\mathcal{I} \subseteq\left(\begin{array}{c}
{[4]} \\
>1
\end{array}\right)} f_{\mathcal{I}}\left(\left(w_{I}\right)_{I \subseteq[4]}\right) .
$$


Then Problem (2) can be written as:

$$
\begin{array}{cl}
\underset{\left(w_{I}\right)_{I \subseteq[4]}}{\operatorname{minimize}} & r \\
\text { subject to } \quad & f_{\mathcal{I}}\left(\left(w_{I}\right)_{I \subseteq[4]}\right) \leq r \text { for all } \mathcal{I} \subseteq\left(\begin{array}{c}
{[4]} \\
>1
\end{array}\right), \\
a_{1}= & w_{1}+w_{2}+w_{3}+w_{4}, \\
a_{2}= & w_{1} w_{2} w_{12}+w_{1} w_{3} w_{13}+w_{1} w_{4} w_{14} \\
& +w_{2} w_{3} w_{23}+w_{2} w_{4} w_{24}+w_{3} w_{4} w_{34}, \\
a_{3}= & w_{1} w_{2} w_{3} w_{12} w_{13} w_{23} w_{123}+w_{1} w_{2} w_{4} w_{12} w_{14} w_{24} w_{124} \\
& +w_{1} w_{3} w_{4} w_{13} w_{14} w_{34} w_{134}+w_{2} w_{3} w_{4} w_{23} w_{24} w_{34} w_{234}, \\
a_{4}= & w_{1} w_{2} w_{3} w_{4} w_{12} w_{13} w_{14} w_{23} w_{24} w_{34} w_{123} w_{124} w_{134} w_{234} w_{1234} .
\end{array}
$$

This resolves all maxima in the objective function at the cost of introducing $2^{11}$ additional constraints, one for each $\mathcal{I} \subseteq\left(\begin{array}{c}{[4]} \\ >1\end{array}\right)$.

Alternatively, one can decompose the space of molecules $\left(\mathbb{R}_{>0}\right)^{2^{4}}$ into $2^{11}$ regions in which either $w_{I} \geq 1$ or $w_{I} \leq 1$ for all $\mathcal{I} \subseteq\left(\begin{array}{c}{[4]} \\ >1\end{array}\right)$. For each $\mathcal{I} \subseteq\left(\begin{array}{c}{[4]} \\ >1\end{array}\right)$, we define a region

$$
\begin{aligned}
O_{\mathcal{I}}:= & \left\{\left(w_{I}\right)_{I \subseteq[n]} \in\left(\mathbb{R}_{>0}\right)^{2^{4}} \mid \text { for all } I \in\left(\begin{array}{c}
{[4]} \\
>1
\end{array}\right): w_{I} \geq 1 \text { if } I \notin \mathcal{I}\right. \text { and } \\
& \left.w_{I} \leq 1 \text { if } I \in \mathcal{I}\right\}
\end{aligned}
$$

so that

$$
\left(\mathbb{R}_{>0}\right)^{2^{4}}=\bigcup_{\mathcal{I} \subseteq\left(\begin{array}{c}
{[4]} \\
>1
\end{array}\right)} O_{\mathcal{I}} \text { and }\|W\|=f_{\mathcal{I}}(W) \text { for } \mathrm{W} \in \mathcal{O}_{\mathcal{I}}
$$

Finding the minimal absolute interaction in $O_{\mathcal{I}}$ hence becomes a straight-forward polynomial optimization problem with few constraints.

$$
\begin{array}{ll}
\underset{\mathrm{W} \in O_{\mathcal{I}}}{\operatorname{minimize}} & f_{\mathcal{I}}(\mathrm{W}) \\
\text { subject to } & \Phi(\mathrm{W})=P
\end{array}
$$

However, this approach requires solving $2^{11}$ polynomial optimization problems, one for each region $\mathcal{O}_{\mathcal{I}}$, which is not easily feasible.

One can reduce the number of regions that require consideration slightly by exploiting the intrinsic symmetry of the problem.

Example 3.1 Consider for example the following polynomial optimization problem:

$$
\begin{array}{ll}
\underset{(x, y) \in \mathbb{R}_{>0}^{2}}{\operatorname{minimize}} & \max \left(x, x^{-1}\right) \cdot \max \left(y, y^{-1}\right) \\
\text { subject to } & a_{1}=x+y, \\
& a_{2}=x \cdot y .
\end{array}
$$

The space $\mathbb{R}_{>0}^{2}$ can be decomposed into 4 regions on which the objective function is $x y, \frac{x}{y}, \frac{y}{x}, \frac{1}{x y}$ respectively, see Fig. 4 . As both objective function and constraints are 


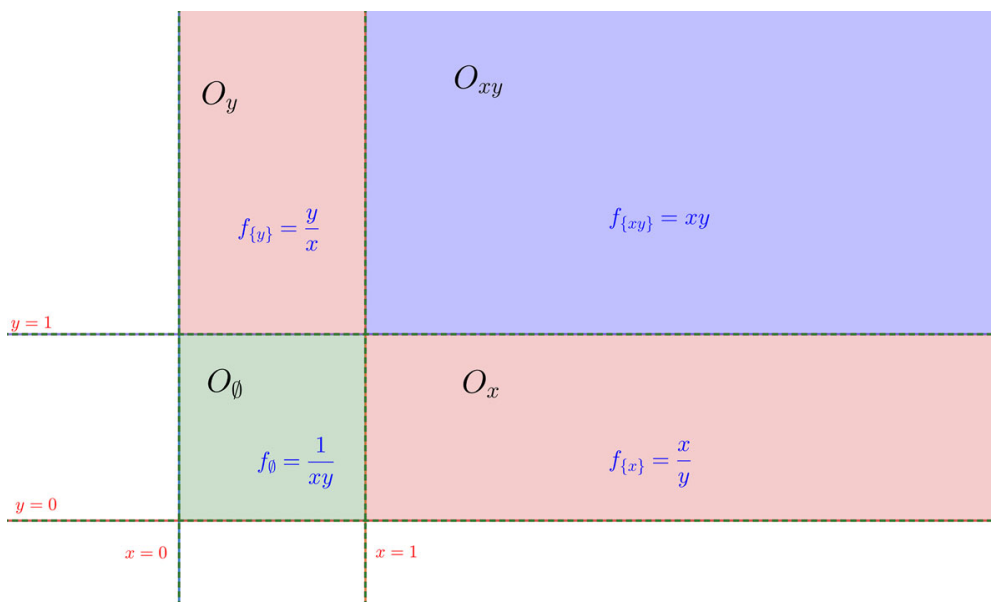

Fig. 4 Partitions in plane

symmetric with respect to permuting $x$ and $y$, the minimum of $f_{\{y\}}$ in $O_{y}$ is same as the minimum of $f_{\{x\}}$ in $O_{x}$. Therefore, it suffices to minimize over three regions instead of all four.

For Problem (2) specifically, this means:

Proposition 3.2 There exists a natural action of the symmetric group $S_{4}$ on the space of molecules $\left(\mathbb{R}_{>0}\right)^{2^{4}}$ by permuting the 4 binding sites:

$$
S_{4} \times\left(\mathbb{R}_{>0}\right)^{2^{4}} \longrightarrow\left(\mathbb{R}_{>0}\right)^{2^{4}}, \quad\left(\sigma,\left(w_{I}\right)\right) \longmapsto \sigma \cdot\left(w_{I}\right):=\left(w_{\sigma(I)}\right)
$$

This action permutes the regions $\mathcal{O}_{\mathcal{I}}$ and preserves binding polynomials as well as absolute interactions, i.e., $\Phi(\sigma \cdot \mathrm{W})=\Phi(\mathrm{W})$ and $\|\sigma \cdot \mathrm{W}\|=\|\mathrm{W}\|$ for all $\mathrm{W} \in\left(\mathbb{R}_{>0}\right)^{2^{4}}$ and all $\sigma \in S_{4}$.

Instead of computing in all $2^{11}$ regions, it therefore suffices to consider one region per $S_{4}$-orbit of regions. However, this still requires 249 regions to be checked, which is still too much for practical purposes.

\subsection{Reducing the degree of the constraints}

Given a binding polynomial $P$, the polynomial equations in Problem (3) arising from its coefficients $a_{1}, \ldots, a_{4}$ pose a serious computational problem. To sidestep this issue we now introduce new coordinates $s_{I}:=\prod_{I^{\prime} \subseteq I} w_{I^{\prime}}$. Here, $s_{I}$ represents the free energy difference of microstate $I$ to the fully unoccupied state. On the positive orthant $\left(\mathbb{R}_{>0}\right)^{2^{n}}$, this defines a bijection 


$$
\begin{array}{cccc}
W=\left(w_{I}\right) & \longmapsto & \left(\prod_{I^{\prime} \subseteq I} w_{I^{\prime}}\right) \\
\uparrow & & n^{\Uparrow} \\
\left(\mathbb{R}_{>0}\right)^{2^{n}} & \longleftrightarrow & \left(\mathbb{R}_{>0}\right)^{2^{n}} \\
\Psi & & \Psi \\
\left(\prod_{I^{\prime} \subseteq I} s_{I^{\prime}}^{(-1)^{\left|I \backslash I^{\prime}\right|}}\right) & \varphi^{-1} & \left(s_{I}\right)=S
\end{array}
$$

In the new coordinates, the formerly polynomial constraints $a_{k}=\sum_{|I|=k} \prod_{I^{\prime} \subseteq I} w_{I^{\prime}}$ become linear constraints $a_{k}=\sum_{|I|=k} s_{I}$ for $k=1, \ldots, n$. While the functions $f_{\mathcal{I}}$ become more complicated in the new coordinates, they still remain monomial and thus it has minimal impact on the runtime: For example, for $\mathcal{I}=\{\{1,2,3\}\}$

$$
f_{\mathcal{I}}(W)=w_{123}^{-1} \cdot \prod_{\substack{|I|>1, I \neq\{1,2,3\}}} w_{I} \text { becomes } f_{\mathcal{I}} \circ \varphi^{-1}(S)=\frac{s_{1234} s_{12}^{2} s_{13}^{2} s_{23}^{2}}{s_{123}^{2} s_{1}^{3} s_{2}^{3} s_{3}^{3} s_{4}} .
$$

In the new coordinates, Problem (3) becomes:

$$
\begin{array}{cl}
\underset{\left(w_{I}\right)_{I \subseteq[4]}}{\operatorname{minimize}} & r \\
\text { subject to } & f_{\mathcal{I}} \circ \varphi^{-1}(S) \leq r \text { for all } \mathcal{I} \subseteq\{I \subseteq[n]|| I \mid>1\}, \\
& a_{1}=s_{1}+s_{2}+s_{3}+s_{4}, \\
& a_{2}=s_{12}+s_{13}+s_{14}+s_{23}+s_{24}+s_{34}, \\
& a_{3}=s_{123}+s_{124}+s_{134}+s_{234}, \\
& a_{4}=s_{1234} .
\end{array}
$$

\subsection{Solving the polynomial optimization problem}

We will use SCIP (Gleixner et al. 2018) to solve polynomial optimization Problem (5), which is currently one of the fastest non-commercial solvers for non-linear programming. It uses a branch and bound method to solve the optimization problem with non-linear constraints. Example 3.3 shows a computation using SCIP for a molecule with 3 binding sites.

Example 3.3 Consider the polynomial $P_{1}$ of Example 2.7. Figure 5 shows the full input on the left and the partial output on the right. In the input, the first constraints $\mathrm{c} 1, \mathrm{c} 2, \mathrm{c} 3$ enforce $\sum_{|I|=k} s_{I}=a_{k}$ for $i=1,2,3$. The remaining constraints $\mathrm{c} 4$ to $\mathrm{c} 19$ enforce $f_{\mathcal{I}} \circ \varphi^{-1}(S) \leq r$. For example, c19 states that $s_{1} s_{2} s_{3} / s_{123} \leq r$ which is equivalent to $f_{\emptyset}(W)=\left(w_{123} w_{12} w_{13} w_{23}\right)^{-1} \leq r$ in the coordinates $w_{I}$. The output states an approximate optimal value of $r=13.5$ and lists all values of $s_{I}$ of the minimal molecule, which gave rise to the numbers in Example 2.7.

Unfortunately, the $2^{11}$ constraints in Problem (5) make it practically impossible to solve directly. Instead, we focus on computing an upper bound $b_{+}$and a lower bound 


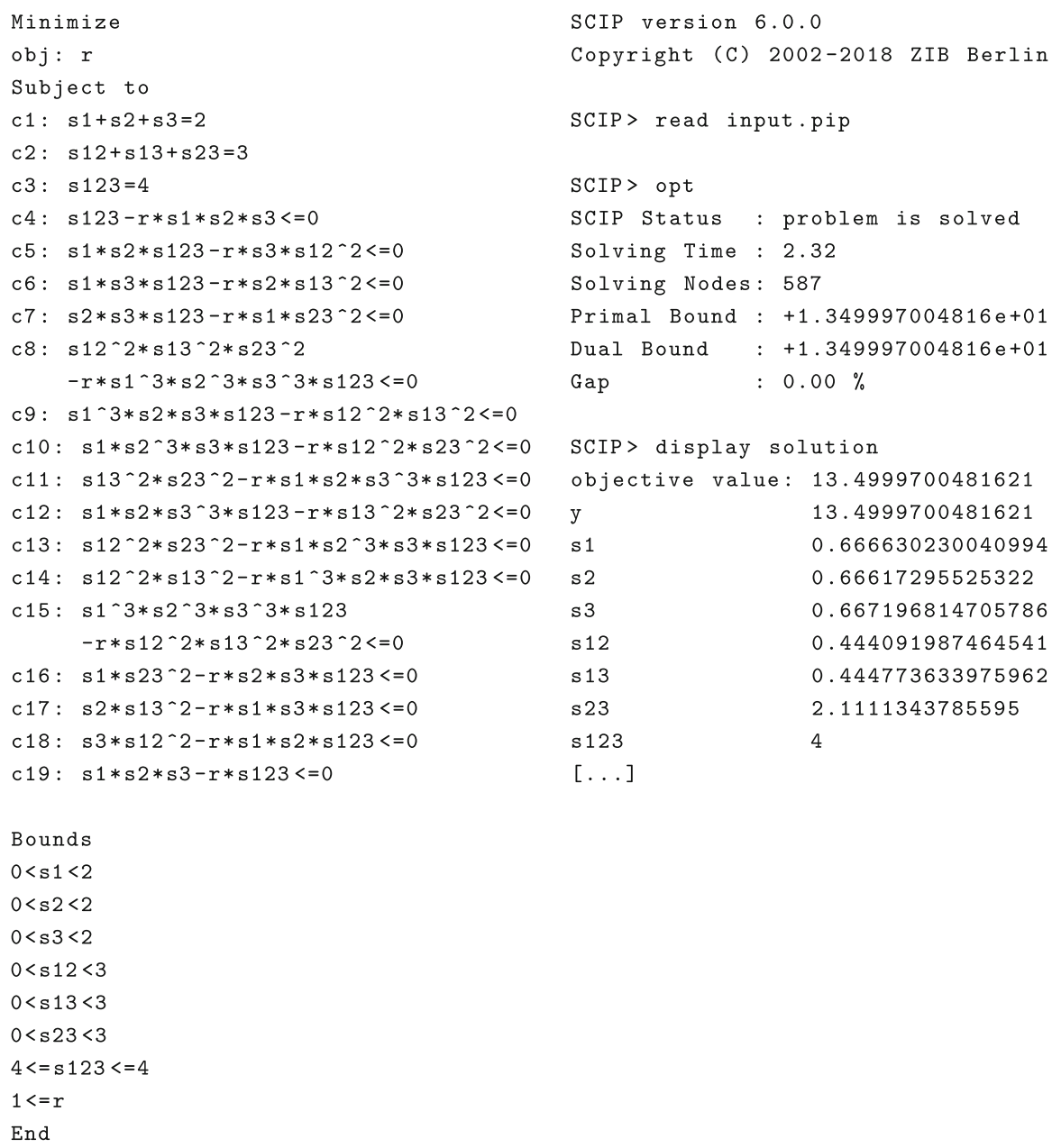

End

Fig. 5 Computing the minimal absolute interaction for a molecule with 3 sites using SCIP

$b_{-}$for the minimal interaction. Both bounds are easier to compute, because they arise from problems with significantly less constraints. Our aim is to obtain bounds which either coincide or are sufficiently close to each other to allow us to compare the different minimal interactions with each other.

\section{The upper bound $b_{+}$}

Our upper bound $b_{+}$for the solution to Problem (5) is the solution to Problem (4) for a region $O_{\mathcal{I}}$. In other words, instead of minimizing the interaction over all molecules in $\left(\mathbb{R}_{>0}\right)^{2^{4}}$, we only minimize it over the molecules in $O_{\mathcal{I}}$. It is an upper bound as the global minimum need not be in the region that we have chosen. Computing the solution to Problem (4) still required changing to the new coordinates $\left(s_{I}\right)$. Our region 
Table 1 Conditions under which binding polynomials 1 to 8 were measured

\begin{tabular}{llll}
\hline Human $\mathrm{Hb}$ & $\begin{array}{l}\text { In the absence } \\
\text { of 2mM DPG }\end{array}$ & $\begin{array}{l}\text { In the presence } \\
\text { of 2mM DPG }\end{array}$ \\
\hline Untreated & $P_{1}$ & $\prec$ & $P_{2}$ \\
& $\curlyvee$ & $\prec$ & $\checkmark$ \\
Treated with iodoacetamide & $P_{3}$ & $\prec$ & $P_{4}$ \\
Treated with $N$-ethylmaeimide & $\curlyvee$ & & $P_{6}$ \\
Treated with carboxypeptidase A & $P_{5}$ & $\prec$ & $\checkmark$ \\
\hline$P_{l} \prec P_{j}$ m & $\curlyvee$ & $P_{7}$ & $P_{8}$ \\
\hline
\end{tabular}

$P_{i} \prec P_{j}$ means $P_{i}$ that the Hill plot of $P_{i}$ has a lower maximal slope compared to $P_{j}$

of choice was $O_{\emptyset}$, i.e., the region where $w_{I} \geq 1$ for all $I \in\left(\begin{array}{c}{[4]} \\ >1\end{array}\right)$, as it was the only region in which all computations terminated successfully during testing.

\section{The lower bound $b_{-}$}

Our lower bound $b_{-}$is the solution to a relaxed Problem (5) with some of the $2^{11}$ constraints of the form $f_{\mathcal{I}} \circ \varphi^{-1}(S) \leq r$ removed. It is a lower bound since removing constraints enlarges the feasible set over which the minimum is computed. Appendix A contains a list of constraints that remain in the final computation. We picked a region $O_{\mathcal{I}}$ in each $S_{4}$ orbit of regions and only considered constraints given by functions $f_{\mathcal{I}}$ that are dominant in one of the regions.

\section{Experimental results}

In this section, we present the results of computational experiments on data from (Imai 1973; Ikeda-Saito et al. 1983) which is also summarized in Connelly et al. (1986). Section 4.1 summarizes the origins of the data, Sect. 4.2 contains the bounds on the minimal interactions, and Sect. 4.3 contains molecules attaining the upper bound.

\subsection{Data}

The first data set consists of eight oxygen binding polynomials $\left(P_{1}\right.$ to $\left.P_{8}\right)$ of human adult hemoglobin in its native form and chemically treated with iodoacetamide, $N$ ethylmaeimide, and carboxypeptidase A. The oxygen binding was observed both in absence and in presence of 2,3-diphosphoglycerate (DPG) (Imai 1973). One of the goals was to determine how the chemical treatments and DPG affect the binding affinity of hemoglobin, the results are indicated by $\succ$ in Table 1 .

The second data set contains five binding polynomials $\left(P_{9}\right.$ to $\left.P_{13}\right)$ of native hemoglobin HbII of Scapharca inaequivalvis measured at different temperatures (Ikeda-Saito et al. 1983, Table 3), see Table 2. One of the goals was to investigate how temperature affects cooperativity in oxygen binding. 
Table 2 Temperature in degree Celsius at which binding polynomials 9-13 were determined

Table 3 Binding polynomials, bounds on the minimal absolute interaction and $n_{\max }$ as reported by Ikeda-Saito et al. (1983), Imai (1973)

\begin{tabular}{cccccc}
\hline Clam HbII & at $10^{\circ}$ & at $15^{\circ}$ & at $20^{\circ}$ & at $25^{\circ}$ & at $30^{\circ}$ \\
\hline$P_{9}$ & $P_{10}$ & $P_{11}$ & $P_{12}$ & $P_{13}$ \\
\hline
\end{tabular}

\begin{tabular}{lllllll}
\hline & $a_{1}$ & $a_{2}$ & $a_{3}$ & $b_{+}$ & $b_{-}$ & $n_{\text {max }}$ \\
\hline$P_{1}$ & 0.835 & 0.379 & 0.541 & 527 & 527 & 2.51 \\
$P_{2}$ & 0.789 & 0.154 & 0.0648 & 3322 & 1600 & 3.09 \\
$P_{3}$ & 1.42 & 2.42 & 0.752 & 111 & 63 & 1.63 \\
$P_{4}$ & 0.647 & 0.568 & 0.0986 & 2002 & 1460 & 2.71 \\
$P_{5}$ & 2.0 & 2.31 & 2.04 & 16 & 16 & 1.44 \\
$P_{6}$ & 0.539 & 0.909 & 0.554 & 3033 & 3030 & 2.27 \\
$P_{7}$ & 3.47 & 4.74 & 2.76 & 2.27 & 1.68 & 1.15 \\
$P_{8}$ & 3.26 & 5.36 & 2.23 & 7.64 & 2.19 & 1.23 \\
$P_{9}$ & 1.4 & 1.0 & 0.62 & 66 & 66 & 2.08 \\
$P_{10}$ & 1.4 & 0.96 & 0.60 & 66 & 66 & 2.10 \\
$P_{11}$ & 1.2 & 0.93 & 0.70 & 123 & 123 & 2.08 \\
$P_{12}$ & 1.4 & 0.95 & 0.62 & 66 & 66 & 2.10 \\
$P_{13}$ & 1.1 & 0.98 & 0.59 & 175 & 175 & 2.12 \\
\hline
\end{tabular}

Coefficients $a_{0}$ and $a_{4}$ are normalized to 1 for all polynomials

Table 3 lists the coefficients $a_{1}, a_{2}$ and $a_{3}$ of the binding polynomials $P_{1}$ to $P_{13}$, the coefficients $a_{0}$ and $a_{4}$ are normalized to 1 . Furthermore, it lists the maximal slope to the Hill plot $n_{\max }$, as reported by Imai (1973) and Ikeda-Saito et al. (1983), which relates the variance of the probability distribution on the macrostates $\{0,1,2,3,4\}$ at the respective ligand activity to the variance of a binomial distribution with the same mean (Abeliovich 2005; Martini et al. 2016a). A value larger than 1 indicates a variance higher than the maximal value an independent system can generate.

\subsection{Minimal absolute interactions}

Table 3 lists the upper $\left(b_{+}\right)$and lower $\left(b_{-}\right)$bounds for the minimal absolute interaction for the binding polynomials $P_{1}$ to $P_{13}$. As explained in Sect. 3.4, solving Problem (5) for $n=4$ is very hard, which is why we computed bounds instead. The upper bound is the minimal absolute interaction in the region $\mathcal{O}_{\emptyset}$, i.e., the minimal interaction in the region where all interactions are 1 or larger. The lower bound comes from a relaxation of Problem (5) which enlarges the set of molecules over which the minimum is computed.

Looking at the bounds $b_{+}$and $b_{-}$in Table 3, we observe two things:

(1) Apart from $P_{2}, P_{3}$, and $P_{8}$, the bounds determine the minimal interaction up to a precision of $15 \%$. 

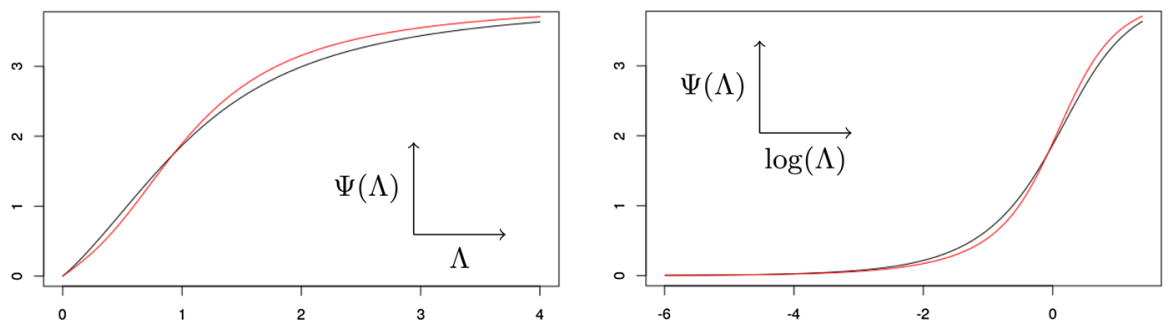

Fig. 6 The binding curves of $P_{9}$ (black) and $P_{11}$ (red) in normal (left) and logarithmic (right) scale (color figure online)

(2) The bounds are almost sufficient to rank the minimal interactions of $P_{1}$ to $P_{8}$ and $P_{9}$ to $P_{13}$ :

$$
\begin{gathered}
\left\|P_{6}\right\|>\left\|P_{4}\right\|>\left\|P_{1}\right\|>\left\|P_{3}\right\|>\left\|P_{5}\right\|>\left\|P_{7}\right\|>\left\|P_{8}\right\| \\
\text { and }\left\|P_{9}\right\|=\left\|P_{10}\right\|=\left\|P_{12}\right\|>\left\|P_{11}\right\|>\left\|P_{13}\right\|
\end{gathered}
$$

with $\left\|P_{2}\right\|$ possibly being larger than $\left\|P_{6}\right\|$ or between $\left\|P_{4}\right\|$ and $\left\|P_{1}\right\|$.

Comparing the ranking of the binding polynomials by their minimal interactions with the ranking by the maximal slope of their Hill plot $n_{\max }$ reveals the following similarities and differences:

\section{$P_{9}$ to $P_{13}$}

We begin by looking at polynomials $P_{9}$ to $P_{13}$ as there are prominent differences between minimal interactions and maximal slopes of the Hill plots: While all maximal slopes are approximately equal, there are stark differences in the minimal interactions. This is because the minimal interaction depends on the entire binding curve, while the maximal slope does not. Figure 6 illustrates that while the binding curves of $P_{9}$ to $P_{13}$ may have identical maximal slopes in logarithmic coordinates, there are subtle differences between both curves. The binding curve of $P_{11}$ has a stronger sigmoidal shape, which is why more interaction is necessary to generate the binding behaviour.

\section{$P_{1}$ to $P_{8}$}

While the ranking by minimal interaction is different to the ranking by maximal slope, we retain nearly all important relations in Table 1, which indicate how the chemicals affect the binding behaviour. The only exceptions are $P_{4}$ and $P_{6}$, where $P_{4}$ has a higher maximal slope and $P_{6}$ has a higher minimal interaction. This is not surprising, as Fig. 7 illustrates that the binding curve $P_{4}$ has a higher maximal slope, while the binding curve $P_{6}$ has a stronger curvature.

Another explanation can be found in the coefficients of $P_{4}$ and $P_{6}$ : In $P_{6}$, coefficient $a_{2}$ is larger than $a_{1}$ and $a_{3}$ and almost same size as $a_{4}=1$. This means that the distribution on the macrostates stabilizes around macrostate 2 . This binding behaviour does not only requires more interaction between the binding sites, it also prevents large 

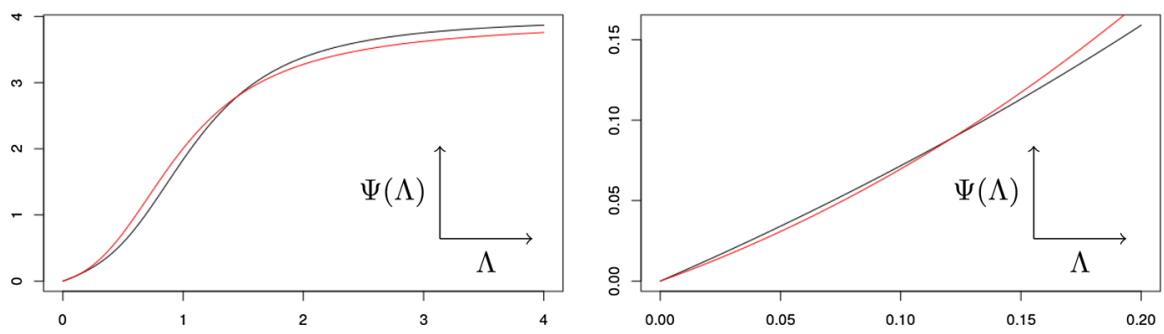

Fig. 7 The binding curves of $P_{4}$ (black) and $P_{6}$ (red) on [0, 4] (left) and zoomed in on [0, 0.2] (right). Note how the red curve starts and ends lower as the black curve, but overtakes it in between (color figure online)

variances of the distribution on the macrostates and thus leads to a reduction of $n_{\max }$. In $P_{4}$, coefficients $a_{1}, a_{2}, a_{3}$ are smaller than $a_{4}=1$. This means that the distribution on the macrostates leans toward macrostate 4 , which leads to a higher value of $n_{\max }$.

\subsection{Molecules solving the optimization problem}

Table 4 lists all molecules realizing the upper bound $b_{+}$of the minimal interaction. We see that the many interaction energies are close to 1 . This is not surprising since we are minimizing the exponential of an $L_{1}$-norm and $L_{1}$ optimizations are known to provide sparse solutions (Tibshirani 1996).

Looking at the structure of the solutions in more detail, we see that most solutions include a non-trivial weight for a pairwise interaction $\left(w_{34}\right)$. The only polynomial whose molecule possesses trivial pairwise interaction is $P_{2}$ which has the highest $n_{\max }$. This is plausible as a high $n_{\max }$ means a high variance of the macroscopic distribution, which in turn implies more weights towards macrostates 0 and 4 . And any nontrivial pairwise interaction would put more weight on macrostate 2 .

Moreover, we mentioned how the macroscopic distribution for $P_{6}$ stabilizes around 2 while the macroscopic distribution for $P_{4}$ leans towards 4 . This can be seen in their minimal molecules in $O_{\emptyset}$, as the molecule for $P_{6}$ has large $w_{34}$ and the molecule for $P_{4}$ has a large $w_{1234}$.

\section{Discussion and outlook}

\subsection{Cooperativity and minimal absolute interaction}

While cooperativity has always been a property of the macroscopic binding behavior, it is often interpreted on a microscopic level, for instance that "binding of a ligand molecule increases the receptor's apparent affinity, and hence increases the chance of another ligand molecule binding" (Stefan and Le Novère 2013). There is however no straightforward connection between the macroscopic and microscopic properties, as generally there are infinitely many different molecules with the same binding curve. Thus creating a bridge between macroscopic and microscopic properties usually relies on additional restrictions, such as having indistinguishable sites. 


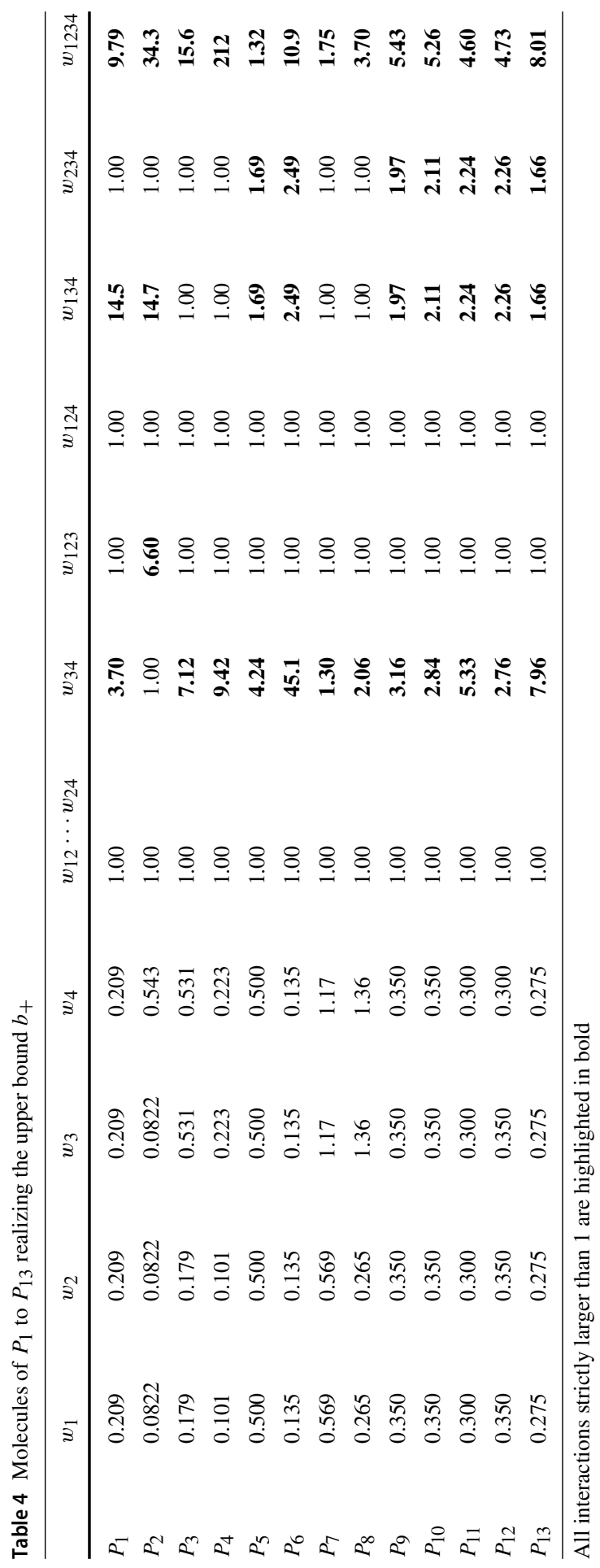


In this paper, we considered the minimal interaction necessary to generate a fixed overall binding curve. In other words, we study the minimal deviation from a molecule with no interaction that is needed to create a prescribed binding behavior. We showed that computing the minimal interaction gives rise to a challenging polynomial optimization problem, and proposed easier ways for obtaining upper and lower bounds. Applying our techniques to overall binding curves and comparing our results to the maximal slope of the Hill plot, we observe:

(1) The upper and lower bounds were sufficient to rank all but one of the curves.

(2) The complete ranking of the curves by minimal interaction may differ from the ranking by maximal Hill slope, but most of the important relations are the same.

(3) In the cases were minimal interaction and maximal Hill slope disagreed, the curve with the higher minimal interaction displayed a stronger curvature (see Figs. 6 and 7).

Conceptually, the minimal interaction considers the entire curve and captures any deviation from an independent system, while the maximal Hill slope only indicates an abnormally high variance of the macroscopic distributions. This is also reflected in our computations, as our results based on the minimal interaction largely coincides with the results based on the maximal Hill slope and the small differences in the results could be explained by signs of interaction outside the region of maximal Hill slope. Additionally, the corresponding minimal molecules may give valuable insights into the microscopic dependencies of the molecule. While the minimal molecule may not be a physically correct representation of the actual chemical molecule that was measured, in a way it describes the simplest possible set of interactions required for the binding curve. Note that we chose to minimize the $L_{1}$-norm of the actual interaction energies because it was the most natural norm in our framework. Alternatively, other $L_{p}$-norms could be used, and it is unclear whether they yield better qualitative results. However, with current methods the minimal interaction is much harder to compute than the maximal Hill slope and thus new mathematical approaches are required.

\subsection{The mathematical problem}

Ligand binding raises many intriguing questions in applied algebra and geometry, and stands to benefit equally from further insights into the intrinsic geometry of the problems. While previous works have explored the application of polynomial system solving techniques to find decoupled molecules in a situation with different types of ligands (Ren et al. 2019; Martini et al. 2013), this work explored the use of algebraic optimization to calculate the minimal interaction required to generate a certain binding behavior.

We illustrated how searching for the minimal interaction energy required to generate a binding curve leads to a problem in algebraic optimization. Even though, computational tools are readily available and the structure of the problem seems relatively simple on first sight, it becomes complicated when more than 3 binding sites are considered. In particular the fact that we had to calculate upper and lower bounds by a relaxed problem and were not able to close their gap completely, shows that our approach is not yet ready for a simple ready-to-use tool to quantify cooperativity. Even 
though we were able to rank the polynomials in Sect. 4.2, we were not able to compute precise minimal energies for all polynomials. We thus believe further research into the computational problem is warranted, though it may need to come from a different direction.

\section{Exploiting symmetries}

One possibility would be to show that the minimal molecule have some symmetry, which would reduce the number of variables (interaction energies) in our problem. While both Problem 5 and the molecules in Table 4 show some kind of symmetry, it is mathematically not clear whether that always needs to be the case. There are results using representation theory which relate symmetries in polynomial optimization problems to symmetries in their solutions (Riener and Safey El Din 2018), however the assumptions on the symmetry in the polynomial optimization problem are quite strong and not satisfied by Problem 5.

\section{Tropical geometry}

Tropical geometry (Maclagan and Sturmfels 2015) revolves around functions on the max-plus semiring, such as the absolute interaction in logarithmic coordinates:

$$
\begin{array}{ccc}
\left(\mathbb{R}_{>0}\right)^{2^{n}} & \longleftrightarrow & \begin{array}{c}
\mathbb{R}^{2^{n}} \\
\Psi
\end{array} \\
\left(w_{I}\right) & \longmapsto & \left(\log \left(w_{I}\right)\right)=:\left(x_{I}\right) \\
\prod_{I \subseteq[n]} \max \left(w_{I}, w_{I}^{-1}\right) & \longmapsto & \longmapsto \\
\sum_{I \subseteq[n]} \max \left(x_{I},-x_{I}\right)=:\left\|\left(x_{I}\right)\right\|_{\text {trop }}
\end{array}
$$

In tropical geometry, the function $\|\cdot\|_{\text {trop }}$ is called a tropical rational function. It is a piecewise-linear function, linear on the image of each $\mathcal{O}_{\mathcal{I}}$, and the boundary separating its regions of linearity is called its tropical hypersurface $T$. Since the gradient of $\|\cdot\|_{\text {trop }}$ is constant on each region of linearity, optimizing $\|\cdot\|_{\text {trop }}$ on any set $L$ boils down to understanding the intersection of $L$ with $T$.

For our problem, $L$ is the image of the feasible set given by the conditions $\Phi(W)=$ $P$ which is an exponential variety of codimension $n$. Theoretically, there exist only a finite number of intersection patterns of $L$ and $T$. Classifying these intersection patterns would allow us to compute minimal interactions and minimal molecules almost instantly.

\section{Tensors}

Tensors (Landsberg 2012) are multidimensional arrays of real numbers which generalize matrices. We can regard molecules as (positive) tensors in $\mathbb{R}^{2 \times \cdots \times 2}=:\left(\mathbb{R}^{2}\right)^{\otimes n}$, whose entries are indexed by $\{0,1\}^{n}$ which is in bijection with the subsets of $[n]$. 
From this point of view, many concepts in tensor theory have natural analogues in ligand binding, such as symmetric tensors and molecules with indistinguishable sites. Notably, a rank one tensors is up to scaling a tensor with entries

$$
(1,0, \ldots): s_{1}, \quad(0,1,0, \ldots): s_{2}, \quad(1,1,0, \ldots): s_{1} s_{2}, \quad \ldots \quad \text { for some } s_{i} \in \mathbb{R}
$$

Hence rank one tensors correspond to molecules with independent sites. Therefore, decomposing a given tensor into rank one tensors translates to decomposing a given molecule into different molecules with independent sites whose mix has the same binding curve. And the tensor rank translates to the minimal number of different molecules with independent sites needed to recreate the same binding curve. Thus the tensor rank of a molecule could serve as an interesting, discrete measure for cooperativity.

\section{Dependency measures}

From a stochastic or statistical point of view, the problem of cooperativity is a problem of quantifying (minimal) dependency, which is a classical research topic in these fields (Rényi 1959; Schweizer and Wolff 1981; Koyak 1987). In particular copulas (Schweizer 1991; Durante and Sempi 2010; Nelsen 2007) have become a common tool to describe dependencies between random variables. Copulas cannot be applied directly to our problem since they are defined on continuous, not discrete, random variables. Moreover, they aim at modeling the dependency of given random variables which in our scenario corresponds to the dependency of the sites of a known molecule. A copula then relates the one-dimensional marginal distributions to the multivariate minimization problem of finding the minimal dependency required to generate a given distribution of a sum of dependent variables is usually not treated. Nevertheless, the problem of cooperativity should also be treated from a more probabilistic point of view and the available concepts should be considered in more detail in the context of ligand binding.

Acknowledgements The authors would like to thank Felipe Serrano for valuable technical support with SCIP. The first and second author were partially supported by the Max Planck Institute for Mathematics in the Sciences. Yue Ren is supported by the UKRI Future Leaders Fellowship (MR/S034463/1). Mohab Safey El Din is supported by the PGMO grant CAMISADO and the European Union's Horizon 2020 research and innovation programme under the Marie Sklodowska-Curie grant Agreement No. 813211 (POEMA).

Open Access This article is licensed under a Creative Commons Attribution 4.0 International License, which permits use, sharing, adaptation, distribution and reproduction in any medium or format, as long as you give appropriate credit to the original author(s) and the source, provide a link to the Creative Commons licence, and indicate if changes were made. The images or other third party material in this article are included in the article's Creative Commons licence, unless indicated otherwise in a credit line to the material. If material is not included in the article's Creative Commons licence and your intended use is not permitted by statutory regulation or exceeds the permitted use, you will need to obtain permission directly from the copyright holder. To view a copy of this licence, visit http://creativecommons.org/licenses/by/4.0/. 


\section{Appendix A. List of constraints}

Below is a compact representation of the list of 180 constraints we considered to compute the lower bounds $b_{-}$of minimal absolute interaction in Fig. 3. There, $\{12,34,123\}$ represents the constraint $f_{\mathcal{I}} \leq r$ for $\mathcal{I}=\{12,34,123\}$, which is the absolute interaction $\|W\|$ for $W \in \mathcal{O}_{\mathcal{I}}$ where $w_{12}, w_{34}, w_{123} \leq 1$ and $w_{I} \geq 1$ otherwise.

$\emptyset,\{12\},\{123\},\{1234\},\{12,13\},\{12,34\},\{12,123\},\{12,134\},\{123,124\},\{12,1234\},\{123,1234\},\{12,13,14\},\{12,13,23\},\{12,13,24\}$, $\{12,13,123\},\{12,13,124\},\{12,34,123\},\{12,13,234\},\{12,123,124\},\{12,123,134\},\{12,134,234\},\{123,124,134\},\{12,13,1234\},\{12$, $34,1234\},\{12,123,1234\},\{12,134,1234\},\{123,124,1234\},\{12,13,14,23\},\{12,13,24,34\},\{12,13,14,123\},\{12,13,23,123\},\{12,13,24$, $123\},\{12,13,23,124\},\{12,13,24,134\},\{12,13,14,234\},\{12,13,123,124\},\{12,13,124,134\},\{12,13,123,234\},\{12,34,123,134\},\{12,34$, $123,124\},\{12,13,124,234\},\{12,123,124,134\},\{12,123,134,234\},\{123,124,134,234\},\{12,13,14,1234\},\{12,13,23,1234\},\{12,13,24$, $1234\},\{12,13,123,1234\},\{12,13,124,1234\},\{12,34,123,1234\},\{12,13,234,1234\},\{12,123,124,1234\}\{12,123,134,1234\},\{12,134$, $234,1234\},\{123,124,134,1234\},\{12,13,14,23,24\},\{12,13,14,23,123\},\{12,13,14,23,124\},\{12,13,24,34,123\},\{12,13,14,23,234\},\{12$, $13,14,123,124\},\{12,13,23,123,124\},\{12,13,24,123,124\},\{12,13,24,123,234\},\{12,13,24,123,134\},\{12,13,23,124,134\},\{12,13,14$, $123,234\},\{12,13,24,134,234\},\{12,13,123,124,134\},\{12,13,123,124,234\},\{12,34,123,124,134\},\{12,13,124,134,234\},\{12,123,124$, $134,234\},\{12,13,14,23,1234\},\{12,13,24,34,1234\},\{12,13,14,123,1234\},\{12,13,23,123,1234\},\{12,13,24,123,1234\},\{12,13,23,124$, $1234\},\{12,13,24,134,1234\},\{12,13,14,234,1234\},\{12,13,123,124,1234\},\{12,13,124,134,1234\},\{12,13,123,234,1234\},\{12,34,123$, $134,1234\},\{12,34,123,124,1234\},\{12,13,124,234,1234\},\{12,123,124,134,1234\},\{12,123,134,234,1234\},\{123,124,134,234,1234\}$, $\{12,13,14,23,24,34\},\{12,13,14,23,24,123\},\{12,13,14,23,24,134\},\{12,13,14,23,123,124\},\{12,13,24,34,123,234\},\{12,13,14,23,124$, $134\},\{12,13,14,23,123,234\},\{12,13,24,34,123,124\},\{12,13,14,23,124,234\},\{12,13,14,123,124,134\},\{12,13,23,123,124,134\},\{12$, $13,24,123,124,134\},\{12,13,14,123,124,234\},\{12,13,24,123,134,234\},\{12,13,23,124,134,234\},\{12,13,123,124,134,234\},\{12,34$, $123,124,134,234\},\{12,13,14,23,24,1234\},\{12,13,14,23,123,1234\},\{12,13,14,23,124,1234\},\{12,13,24,34,123,1234\},\{12,13,14$, $23,234,1234\},\{12,13,14,123,124,1234\},\{12,13,23,123,124,1234\},\{12,13,24,123,124,1234\},\{12,13,24,123,234,1234\},\{12,13,24$, $123,134,1234\},\{12,13,23,124,134,1234\},\{12,13,14,123,234,1234\},\{12,13,24,134,234,1234\},\{12,13,123,124,134,1234\},\{12,13$, $123,124,234,1234\},\{12,34,123,124,134,1234\},\{12,13,124,134,234,1234\},\{12,123,124,134,234,1234\},\{12,13,14,23,24,34,123\}$, $\{12,13,14,23,24,123,124\},\{12,13,14,23,24,123,134\},\{12,13,14,23,24,134,234\},\{12,13,14,23,123,124,134\},\{12,13,14,23,123,124$, $234\},\{12,13,24,34,123,124,134\},\{12,13,14,23,124,134,234\},\{12,13,14,123,124,134,234\},\{12,13,23,123,124,134,234\},\{12,13,24$, $123,124,134,234\},\{12,13,14,23,24,34,1234\},\{12,13,14,23,24,123,1234\},\{12,13,14,23,24,134,1234\},\{12,13,14,23,123,124,1234\}$, $\{12,13,24,34,123,234,1234\},\{12,13,14,23,124,134,1234\},\{12,13,14,23,123,234,1234\},\{12,13,24,34,123,124,1234\},\{12,13,14,23$, $124,234,1234\},\{12,13,14,123,124,134,1234\},\{12,13,23,123,124,134,1234\},\{12,13,24,123,124,134,1234\},\{12,13,14,123,124,234$, $1234\},\{12,13,24,123,134,234,1234\},\{12,13,23,124,134,234,1234\},\{12,13,123,124,134,234,1234\},\{12,34,123,124,134,234,1234\}$, $\{12,13,14,23,24,34,123,124\},\{12,13,14,23,24,123,124,134\},\{12,13,14,23,24,123,134,234\},\{12,13,14,23,123,124,134,234\},\{12,13$, $24,34,123,124,134,234\},\{12,13,14,23,24,34,123,1234\},\{12,13,14,23,24,123,124,1234\},\{12,13,14,23,24,123,134,1234\},\{12,13,14$, $23,24,134,234,1234\},\{12,13,14,23,123,124,134,1234\},\{12,13,14,23,123,124,234,1234\},\{12,13,24,34,123,124,134,1234\},\{12,13$, $14,23,124,134,234,1234\},\{12,13,14,123,124,134,234,1234\},\{12,13,23,123,124,134,234,1234\},\{12,13,24,123,124,134,234,1234\}$, $\{12,13,14,23,24,34,123,124,134\},\{12,13,14,23,24,123,124,134,234\},\{12,13,14,23,24,34,123,124,1234\},\{12,13,14,23,24,123,124$, $134,1234\},\{12,13,14,23,24,123,134,234,1234\},\{12,13,14,23,123,124,134,234,1234\},\{12,13,24,34,123,124,134,234,1234\},\{12$, $13,14,23,24,34,123,124,134,234\},\{12,13,14,23,24,34,123,124,134,1234\},\{12,13,14,23,24,123,124,134,234,1234\},\{12,13,14,23$, $24,34,123,124,134,234,1234\}$

\section{References}

Abeliovich H (2005) An empirical extremum principle for the hill coefficient in ligand-protein interactions showing negative cooperativity. Biophys J 89(1):76-79

Abeliovich H (2016) On hill coefficients and subunit interaction energies. J Math Biol 73(6-7):1399-1411 
Adair GS, Bock AV, Jr HF (1925) The hemoglobin system VI. The oxygen dissociation curve of hemoglobin. J Biol Chem 63(2):529-545

Barcroft J (1913) The combinations of haemoglobin with oxygen and with carbon monoxide. II. Biochem J 7(5):481

Ben-Naim AY (2013) Cooperativity and regulation in biochemical processes. Springer, Berlin

Bohr C, Hasselbalch K, Krogh A (1904) Ueber einen in biologischer beziehung wichtigen einfluss, den die kohlensäurespannung des blutes auf dessen sauerstoffbindung übt 1. Skandinavisches Archiv für Physiologie 16(2):402-412

Connelly P, Robert C, Briggs W, Gill S (1986) Analysis of zeros of binding polynomials for tetrameric hemoglobins. Biophys Chem 24(3):295-309

Durante F, Sempi C (2010) Copula theory: an introduction. In: Jaworski P et al (eds) Copula theory and its applications. Springer, Berlin, pp 3-31

Gleixner A, Bastubbe M, Eifler L, Gally T, Gamrath G, Gottwald RL, Hendel G, Hojny C, Koch T, Lübbecke ME, Maher SJ, Miltenberger M, Müller B, Pfetsch ME, Puchert C, Rehfeldt D, Schlösser F, Schubert C, Serrano F, Shinano Y, Viernickel JM, Walter M, Wegscheider F, Witt JT, Witzig J (2018) The SCIP Optimization Suite 6.0. Technical report, Optimization Online. http://www.optimization-online.org/ DB_HTML/2018/07/6692.html

Greuet A, Safey El Din M (2014) Probabilistic algorithm for polynomial optimization over a real algebraic set. SIAM J Optim 24(3):1313-1343

Gutierrez P, Monteoliva D, Diambra L (2009) Role of cooperative binding on noise expression. Phys Rev E 80(1):011914

Hill AV (1913) The combinations of haemoglobin with oxygen and with carbon monoxide. I. Biochem J $7(5): 471$

Hill TL (1985) Cooperativity theory in biochemistry: steady-state and equilibrium systems. Springer series in molecular and cell biology. Springer, New York

Hunter CA, Anderson HL (2009) What is cooperativity? Angew Chem Int Ed 48(41):7488-7499

Ikeda-Saito M, Yonetani T, Chiancone E, Ascoli F, Verzili D, Antonini E (1983) Thermodynamic properties of oxygen equilibria of dimeric and tetrameric hemoglobins from scapharca inaequivalvis. J Mol Biol 170(4):1009-1018

Imai K (1973) Analyses of oxygen equilibriums of native and chemically modified human adult hemoglobins on the basis of adir's stepwise oxygenation theory and the allosteric model of monod, wyman, and changeux. Biochemistry 12(5):798-808

Koyak RA et al (1987) On measuring internal dependence in a set of random variables. Ann Stat 15(3):12151228

Landsberg JM (2012) Tensors: geometry and applications. Graduate studies in mathematics, vol 128. American Mathematical Society, Providence

Lasserre J (2001) Global optimization with polynomials and the problem of moments. SIAM J Optim 11(3):796-817

Lenaerts T, Ferkinghoff-Borg J, Schymkowitz J, Rousseau F (2009) Information theoretical quantification of cooperativity in signalling complexes. BMC Syst Biol 3(1):9

Maclagan D, Sturmfels B (2015) Introduction to tropical geometry. Graduate studies in mathematics, vol 161. American Mathematical Society, Providence

Martini JWR (2017) A measure to quantify the degree of cooperativity in overall titration curves. J Theor Biol 432:33-37

Martini JWR, Ullmann GM (2013) A mathematical view on the decoupled sites representation. J Math Biol 66(3):477-503

Martini JWR, Schlather M, Ullmann GM (2013) On the interaction of different types of ligands binding to the same molecule part ii: systems with $\mathrm{n}$ to 2 and $\mathrm{n}$ to 3 binding sites. J Math Chem 51(2):696-714

Martini JWR, Diambra L, Habeck M (2016a) Cooperative binding: a multiple personality. J Math Biol 72(7):1747-1774

Martini JWR, Schlather M, Schütz S (2016b) A model for carrier-mediated biological signal transduction based on equilibrium ligand binding theory. Bull Math Biol 78(5):1039-1057

Monteoliva D, McCarthy CB, Diambra L (2013) Noise minimisation in gene expression switches. PLoS ONE 8(12):e84020

Nelsen RB (2007) An introduction to copulas. Springer, Berlin 
Onufriev A, Ullmann GM (2004) Decomposing complex cooperative ligand binding into simple components: connections between microscopic and macroscopic models. J Phys Chem B 108(30):1115711169

Onufriev A, Case DA, Ullmann GM (2001) A novel view of ph titration in biomolecules. Biochemistry 40(12):3413-3419

Parrilo PA (2003) Semidefinite programming relaxations for semialgebraic problems. Math Program 96(2):293-320

Ren Y, Martini JWR, Torres J (2019) Decoupled molecules with binding polynomials of bidegree (n,2). J Math Biol 78(4):879-898. https://doi.org/10.1007/s00285-018-1295-x

Rényi A (1959) On measures of dependence. Acta Math Hung 10(3-4):441-451

Riener C, Safey El Din M (2018) Real root finding for equivariant semi-algebraic systems. In: ISSAC'18Proceedings of the 2018 ACM international symposium on symbolic and algebraic computation. ACM, New York, pp 335-342

Rong C, Zhao D, Zhou T, Liu S, Yu D, Liu S (2019) Homogeneous molecular systems are positively cooperative, but charged molecular systems are negatively cooperative. J Phys Chem Lett 10(8):17161721. https://doi.org/10.1021/acs.jpclett.9b00639

Roy RD, Rosenmund C, Stefan MI (2017) Cooperative binding mitigates the high-dose hook effect. BMC Syst Biol 11(1):74

Salakhieva DV, Sadreev II, Chen MZ, Umezawa Y, Evstifeev AI, Welsh GI, Kotov NV (2016) Kinetic regulation of multi-ligand binding proteins. BMC Syst Biol 10(1):32

Schellman JA (1975) Macromolecular binding. Biopolymers 14(5):999-1018

Schweizer B (1991) Thirty years of copulas. In: Kotz S et al (eds) Advances in probability distributions with given marginals. Springer, Berlin, pp 13-50

Schweizer B, Wolff EF (1981) On nonparametric measures of dependence for random variables. Ann Stat 9(4):879-885

Stefan MI, Le Novère N (2013) Cooperative binding. PLoS Comput Biol 9(6):e1003106

Tibshirani R (1996) Regression shrinkage and selection via the lasso. J Roy Stat Soc Ser B (Methodol) 58(1):267-288

Wyman J, Gill SJ (1990) Binding and linkage: functional chemistry of biological macromolecules. University Science Books, Mill Valley

Publisher's Note Springer Nature remains neutral with regard to jurisdictional claims in published maps and institutional affiliations. 